Löffelholz, Martin; Auer, Claudia; Schleicher, Kathrin:

\title{
Vorsichtige Annäherung : die Beziehungen der Bundeswehr zu den Medien vom Ende des Kalten Krieges bis heute
}

\footnotetext{
Zuerst erschienen in: Militärgeschichtliche Zeitschrift / Zentrum für Militärgeschichte und Sozialwissenschaften der Bundeswehr . - Berlin : de Gruyter. - 70 (2011), 1, p. 69-84.

Erstveröffentlichung: 2011-12-01

ISSN (online): $\quad$ 2196-6850

ISSN (print): $\quad$ 2193-2336

DOI: $\quad$ 10.1524/mgzs.2011.0006

[Zuletzt gesehen: 2019-07-26]
}

„Im Rahmen der hochschulweiten Open-Access-Strategie für die Zweitveröffentlichung identifiziert durch die Universitätsbibliothek IImenau."

"Within the academic Open Access Strategy identified for deposition by IImenau University Library."

„Dieser Beitrag ist mit Zustimmung des Rechteinhabers aufgrund einer (DFGgeförderten) Allianz- bzw. Nationallizenz frei zugänglich."

"This publication is with permission of the rights owner freely accessible due to an Alliance licence and a national licence (funded by the DFG, German Research Foundation) respectively."

\section{DFG}

Nationallizenzen 


\section{Vorsichtige Annäherung. Die Beziehungen der Bundeswehr zu den Medien vom Ende des Kalten Krieges bis heute}

Seit dem Ende der Ost-West-Konfrontation spielen Öffentlichkeit, Journalismus und Public Relations im sicherheitspolitischen Diskurs in Deutschland unverkennbar eine größere Rolle. Für die Bundeswehr signalisierte insbesondere die Auflösung der >Akademie für Psychologische Verteidigung « in Waldbröl und die anschließende Gründung der >Akademie der Bundeswehr für Information und Kommunikation` (AIK) im März 1990 in Strausberg ein Umdenken - von einem Gegeneinander von Militär und Öffentlichkeit zu einem stärkeren Miteinander ${ }^{1}$. Mit der zunehmenden Beteiligung an Auslandseinsätzen und dem damit verbundenen erhöhten Informationsbedarf der Bevölkerung verstärkte sich diese neue Sicht. Vor allem mit der Mitwirkung an der UN-Mission in Somalia (1993/94) wuchs das öffentliche und damit mediale Interesse an der Bundeswehr und dem für Auslandseinsätze zuständigen Bundesministerium der Verteidigung (BMVg). Mit der Beteiligung an den NATO-Einsätzen im ehemaligen Jugoslawien (ab 1995), der militärischen Intervention im Kosovo (1999) und dem Engagement deutscher Streitkräfte in Afghanistan seit 2001 stiegen zudem die Anforderungen an die kommunikative Auseinandersetzung mit gegnerischen Truppen, an die zielgruppengerechte Information der Bevölkerung in Einsatzgebieten und an die politische Legitimation militärischer Operationen in der deutschen Öffentlichkeit.

Während das BMVg und die Bundeswehr ihren Beziehungen zu den Medien mit dem Ziel der öffentlichen Legitimation von Auslandseinsätzen deshalb seit Beginn der 1990er Jahre eine neue Ausrichtung gaben, haben sich die kommunikationswissenschaftliche und historische Forschung bislang kaum um militärische Medienbeziehungen gekümmert ${ }^{2}$. Wissenschaftliche Studien konzentrierten sich bisher vor allem auf die Rolle der Medien in Kriegen³, während das Militär und

$1 \quad$ Vgl. Oliver Zöllner, Dialog als kommunikative Strategie. Öffentlichkeitsarbeit staatlicher Stellen am Beispiel der Akademie der Bundeswehr für Information und Kommunikation, Waldbröl 1993.

2 Zu den wenigen Studien gehören Thorsten Loch, Das Gesicht der Bundeswehr. Kommunikationsstrategien in der Freiwilligenwerbung der Bundeswehr 1956-1989, München 2008 (= Sicherheitspolitik und Streitkräfte der Bundesrepublik Deutschland, 8); Patrick James Hofmann, Die Pressearbeit der Bundeswehr. Öffentlicher Diskurs, interne Organisation und Fallstudienanalyse in der Vor- und Anfangsphase (1950-1963), Magisterarbeit Universität Leipzig 1999; Franz Dörner, Das Verhältnis zwischen Massenmedien und Bundeswehr. Eine empirische Untersuchung, Diss. Mainz 1991; Joachim Kannicht, Die Bundeswehr und die Medien. Material zur Presse- und Öffentlichkeitsarbeit in Verteidigungsfragen, Regensburg 1982 (= Die Bundeswehr. Eine Gesamtdarstellung, 14); Wilfried von Bredow, Der Primat militärischen Denkens: die Bundeswehr und das Problem der okkupierten Öffentlichkeit, Köln 1969.

3 Vgl. u.a. Thomas Dominikowski, Massenmedien und Massenkrieg. Historische Annäherung an eine unfriedliche Symbiose. In: Krieg als Medienereignis, Bd 2: Krisenkommunikation im 21. Jahrhundert. Hrsg. von Martin Löffelholz, Wiesbaden 2004, S. 59-80; Nach dem Golf-/Krieg ist vor dem Golf-/Krieg. Zur Militarisierung der Wahrnehmung in den Massenmedien. Hrsg. von Heinz-B. Heller, Marburg 2009; Philip Hammond, »Hu- 
seine Beziehungen zu den Medien nahezu ausgeblendet wurden. Lediglich Medienstrategien des US-Militärs wurden gelegentlich thematisiert, ohne dabei jedoch empirisch vorzugehen ${ }^{4}$. Sogar die Militärsoziologie, die sich ausdrücklich dem Militär als Forschungsgegenstand zuwendet, hat sich bislang nicht dezidiert mit den Beziehungen von Streitkräften und Medien beschäftigt ${ }^{5}$. Begründet wird diese Zurückhaltung mit dem Einfluss, den das Militär auf die gegenwartsbezogene Forschung ausübt: »Aufgrund der politischen Sensibilität der Ergebnisse bzw. aus Angst vor deren Instrumentalisierung (durch die Medien, den politischen Gegner) reguliert das Militär den Zugang zum Forschungsgegenstand ${ }^{6}$. « Betroffen davon sind jene wissenschaftlichen Untersuchungen, deren Erkenntnisinteressen auf die Gegenwart bzw. die letzten drei Jahrzehnte gerichtet sind, denn ministerielle Akten unterliegen einer Einsichtssperre von 30 Jahren. Zudem wird der Zugang von Forschern zu militärischen Organisationen und zum Ministerium aktiv kontrolliert ${ }^{7}$.

»Wie viele andere Institutionen und Organisationen setzt auch das Militär einer sozialwissenschaftlichen Betrachtung manchmal Widerstand entgegen. Er ist in den Streitkräften noch besonders ausgeprägt, da das dort vorhandene Geheimhaltungsgebot nicht selten über die unmittelbar sicherheitsempfindlichen Bereiche hinaus ausgedehnt wird ${ }^{8} . \ll$

manitäre Intervention « und »Krieg gegen den Terror«. Das Verhalten der Medien vom Kosovo bis zum Irak. In: Krieg als Medienereignis, Bd 2 (wie Anm. 4), S. 99-120; David R. Willcox, Propaganda, the press and conflict: the Gulf War and Kosovo, London 2005; Jörg Becker, Afghanistan: der Krieg und die Medien. In: Jahrbuch für Friedens- und Konfliktforschung, Baden-Baden 2002, S. 142-172.

4 Vgl. Magnus-Sebastian Kutz, Die US-amerikanische Öffentlichkeitsarbeit zur Rechtfertigung des Irak-Krieges im Kontext neokonservativer Ideologie. In: Der Irak-Konflikt in den Medien. Eine sprach-, politik- und kommunikationswissenschaftliche Analyse. Hrsg. von Una Dirks, Konstanz 2010, S. 41-63; Andrea Szukala, Informationsoperationen und die Fusion militärischer und medialer Instrumente in den USA. Der Versuch einer militärischen Antwort auf die neuen Bedrohungen. In: Medien \& Kommunikationswissenschaft, 53 (2005), 2/3, S. 222-240; Walter Jertz und Carsten Bockstette, Strategisches Informationsmanagement. Informations- und Öffentlichkeitsarbeit aus militärischer Perspektive. In: Krieg als Medienereignis, Bd 2 (wie Anm. 3), S. 215-237; Carsten Schlüter, Information Operations. Die Weiterentwicklung US-militärischer Strategien zur Instrumentalisierung der Medien. In: Krieg als Medienereignis, Bd 2 (wie Anm. 3), S. 239-254; Thymian Bussemer, Medien als Kriegswaffe. Eine Analyse der amerikanischen Militärpropaganda im Irak-Krieg. In: Aus Politik und Zeitgeschichte, 49-50/2003, S. 20-28; Tile von Damm, Die Öffentlichkeitsarbeit der Bundeswehr - die Truppe als modernes Promotion- und Marketingunternehmen. In: Medien zwischen Krieg und Frieden. Hrsg. von Ulrich Albrecht und Jörg Becker, Baden-Baden 2002, S. 55 f.

5 Vgl. Nina Leonhard und Ines-Jaqueline Werkner, Militär als Gegenstand der Forschung. In: Militärsoziologie. Eine Einführung. Hrsg. von Nina Leonhard und Ines-Jaqueline Werkner, Wiesbaden 2005, S. 13-23.

6 Leonhard/Werkner, Militär als Gegenstand der Forschung (wie Anm. 5), S. 16.

7 Zu den Zugangschwierigkeiten zum Militär als wissenschaftlicher Untersuchungsgegenstand vgl. Claudia Auer und Kathrin Schleicher, Analyse, Evaluation, Kritik: Wie eine theoriegeleitete kommunikationswissenschaftliche Studie einer Großorganisation nützt. In: Theoretisch praktisch!? Anwendungsoptionen und gesellschaftliche Relevanz der Kommunikations- und Medienforschung. Hrsg. von Susanne Fengler, Tobias Eberwein und Julia Jorch, Konstanz 2012 (in Vorbereitung).

8 Paul Klein, Werner Kriesel und Ekkehard Lippert, Militär und Gesellschaft. Bibliographie zur Militärsoziologie 1979-1997, Strausberg 1997, S. 16. 
Vor diesem Hintergrund kommt dem hier vorstellten Forschungsprojekt, das seit Mai 2009 von der Deutschen Forschungsgemeinschaft (DFG) für einen Zeitraum von drei Jahren gefördert wird, besondere Bedeutung zu. Denn nach einer aufwendigen Diskussions- und Vorbereitungsphase haben BMVg und Bundeswehr erstmals detailliert Einblicke in die Ziele, Strukturen und Herausforderungen militärischer Medienbeziehungen gewährt. Die Studie gehört zum Forschungsprojekt >Militärische Media Relations`, das aus zwei Teiluntersuchungen besteht: (1) die Beziehungen der Bundeswehr zu den Medien und (2) die Beziehungen der USStreitkräfte ${ }^{9}$ zu den Medien. Im Kern geht es in dem Gesamtprojekt um die Frage, wie die Beziehungen von Streitkräften und Medien seit 1990 gestaltet und gegebenenfalls verändert wurden. Der vorliegende Beitrag präsentiert Kernergebnisse des ersten Teilprojekts zu den Medienbeziehungen der Bundeswehr.

Das als Beginn des Untersuchungszeitraums ausgewählte Ende des Kalten Krieges 1989/90 bedeutete für die Bundeswehr eine Zäsur: Das sicherheitspolitische Umfeld veränderte sich radikal, und mit dem Beitritt der DDR zur Bundesrepublik musste die ehemalige NVA in die bundesdeutschen Streitkräfte integriert werden. Zudem wird die Bundeswehr - ein weiteres Novum - seit 1990 im Rahmen friedenserhaltender Maßnahmen außerhalb der Bundesrepublik Deutschland eingesetzt. Schon mit der Beteiligung an der UN-Mission in Somalia in den Jahren 1993/94 begann eine grundlegende Neuausrichtung der Truppe hin zu einer Einsatzarmee - mit gravierenden Konsequenzen auch für die militärischen Medienbeziehungen. Als Ende des Untersuchungszeitraums haben wir 2011 gesetzt: Die interessante Frage, wie sich der Rücktritt des ehemaligen Verteidigungsministers Karl-Theodor zu Guttenberg auf die Medienbeziehungen der Bundeswehr auswirkt, werden wir allerdings erst in einem späteren Beitrag behandeln können.

Obgleich die Umwälzungen der Jahre 1989/90 für die Bundeswehr äußerst bedeutsam waren, wurden die Uhren aber damals selbstverständlich nicht auf Null gestellt. Die Entwicklung der militärischen Medienbeziehungen nach 1990 beruht nicht zuletzt auf Kontinuitäten und Traditionen, deren Wurzeln in der Zeit des OstWest-Konflikts liegen. Umfassende Studien zu den Beziehungen von Bundeswehr und Medien in den Jahren zwischen 1955 und 1990 stehen zwar noch aus. Einzelne Arbeiten ermöglichen jedoch zumindest Einblicke in das keineswegs spannungsfreie Verhältnis von Streitkräften und Medien in dieser Zeit:

»Die Häufigkeit und Stärke der Animositäten vonseiten der Truppe hat nun aber in den sechziger Jahren eher zu- als abgenommen. Weite Kreise der Truppe missverstehen Kritik und Misstrauen der Publikationsmittel. Ihre Reaktionen erfolgen prompt und krass: sie wollen mit der Presse nichts mehr zu tun haben ${ }^{10}$.« Ein Presseoffizier sei insofern eher als »Presse-Abwehr-Offizier ${ }^{11} \mathrm{zu}$ charakterisieren, wie von Bredow 1969 schreibt. Ähnlich kritisch sehen andere Autoren die Medienbeziehungen der Bundeswehr bis in die 1980er Jahre ${ }^{12}$. In den siebziger Jah-

9 Das Militär der USA, welches wir als Vergleichsgegenstand gewählt haben, dient Streitkräften weltweit als Referenz, auch der Bundeswehr - keineswegs apologetisch, oftmals sogar dezidiert abgrenzend. Das ändert aber nichts an der zentralen Rolle, welche die US-Streitkräfte für die Bundeswehr sowohl militärstrategisch, als auch als unmittelbarer Kooperationspartner im Rahmen der NATO besitzen.

10 Bredow, Der Primat militärischen Denkens (wie Anm. 2), S. 64.

11 Ebd.

12 Dörner, Das Verhältnis zwischen Massenmedien und Bundeswehr (wie Anm. 2); Kannicht, Die Bundeswehr und die Medien (wie Anm. 2). 
ren habe es zwar Bemühungen um eine professionellere Gestaltung der Presseund Informationsarbeit gegeben, etwa die Gründung des Leitungsstabs der Presseund Informationsarbeit oder die damals neu eingerichteten Stellen der hauptamtlichen Presseoffiziere. Gleichzeitig charakterisierten jedoch Misstrauen und Missverständnisse die Beziehungen von Bundeswehr und Medien: »Die Geheimniskrämerei in vielen Kommandostellen unserer Armee ist nicht nur grotesk, sie ist auch gefährlich. Sie erzeugt Misstrauen bei den Journalisten, und Misstrauen erzeugt Missverständnisse, Abneigung, Voreingenommenheit « ${ }^{13}$, wie Kannicht in seinem 1982 erschienenen Werk einen Kommandeur zitiert. In einer Längsschnittanalyse des Verhältnisses von Bundeswehr und Fernsehen kommen Bleicher und Hickethier ${ }^{14}$ zu der zusammenfassenden Einschätzung, dass "nur langfristig und auf eine eher verhaltene Weise eine Normalisierung des anfangs gespannten Verhältnisses von Bundeswehr und Fernsehen festzustellen « ${ }^{15}$ sei. In den siebziger Jahren hätten Bundeswehr und Medien sich eher skeptisch gegenüber gestanden. In den achtziger Jahren habe es verstärkt Kooperationen zwischen Bundeswehr und öffentlich-rechtlichem Fernsehen gegeben. In den neunziger Jahren sei es »zur >Normalisierung « des Verhältnisses von Bundeswehr und Medien « ${ }^{16}$ gekommen.

Um unter anderem diese Einschätzung zu prüfen, wurde ein MehrmethodenDesign aus Dokumentenanalyse ${ }^{17}$ und Leitfadeninterviews ${ }^{18}$ eingesetzt. Beide Methoden gehören zu den Standardverfahren der sozialwissenschaftlichen Organisationsanalyse $^{19}$. Da der Studie ein exploratives Erkenntnisziel zugrunde liegt, wird

13 Kannicht, Die Bundeswehr und die Medien (wie Anm. 2), S. 39.

14 Joan K. Bleicher und Knut Hickethier, Der Blick des Fernsehens auf die Bundeswehr. In: Die Bundeswehr 1955 bis 2005: Rückblenden, Einsichten, Perspektiven. Im Auftrag des MGFA hrsg. von Frank Nägler, München 2007 (=Sicherheitspolitik und Streitkräfte der Bundesrepublik Deutschland, 7), S. 269-290.

15 Bleicher/Hickethier, Der Blick des Fernsehens auf die Bundeswehr (wie Anm. 14), S. 270.

16 Ebd., S. 286.

17 Auf Basis eines detaillierten Codebuchs wurden rund 500 Dokumente analysiert, die zwischen 1990 und 2011 von den zuständigen Ministerien (BMVg, Pentagon) oder den jeweiligen militärischen Organisationen (Bundeswehr, U.S. Armed Forces) herausgegeben wurden und sich auf die militärische Medienarbeit beziehen.

18 Das Leitfadengespräch ist eine standardisierte Befragung und damit »eine besondere Form der geplanten Kommunikation, die auf einem Fragebogen basiert. Ihr Ziel ist es, zahlreiche individuelle Antworten zu generieren, die in ihrer Gesamtheit zur Klärung einer (wissenschaftlichen) Fragestellung beitragen.«(Wiebke Möhring und Daniela Schlütz, Die Befragung in der Medien- und Kommunikationswissenschaft, Wiesbaden 2003, S. 14) Der Leitfaden gewährleistet dabei die Vergleichbarkeit der Ergebnisse. Bei der vorgenommenen Befragung wurden 50 teilstrukturierte Leitfadengespräche mit Funktionsträgern aus BMVg und Bundeswehr geführt, die zwischen 1990 bis 2011 in diesen Bereichen tätig waren. Die ausgewählten Personen repräsentieren verschiedene Hierarchieebenen sowie alle relevanten funktionalen Bereiche militärischer Medienbeziehungen (Public Relations, Nachwuchswerbung, Öffentlichkeitsarbeit, Operative Information, militärisches Nachrichtenwesen, Militär und Unterhaltungsindustrie). Berücksichtigt wurden zudem die politische und militärische Führungsebene sowie Spezialisten für Kommunikation wie etwa Pressesprecher der zuständigen Ministerien oder Leiter militärischer Pressestellen. Die (noch andauernde) Auswertung der Gespräche liefert vielfältige Einblicke in die Gestaltung militärischer Medienbeziehungen, insbesondere zur Umsetzung normativer Vorgaben, zu formalisierten und informellen Strukturen sowie zu den Leistungen der militärischen Medienarbeit.

19 Vgl. Lutz von Rosenstiel, Organisationsanalyse. In: Qualitative Forschung. Ein Handbuch. Hrsg. von Uwe Flick, Ernst von Kardorff und Ines Steinke, Reinbek bei Hamburg 2008, S. 231 und S. 233. 
eine induktive Forschungslogik verfolgt, bei der von einzelnen Beobachtungen auf generelle Aussagen geschlossen wird ${ }^{20}$. Die Analyse stützt sich auf die Wirklichkeitsdefinitionen ${ }^{21}$, Sozialisationen und Deutungsmuster der Befragten ${ }^{22}$, deren Aussagen zu einer Version der sozialen Welt führen, die als viabel angesehen wird, solange sie nicht durch weitere Forschungen entkräftet wird. Als illustrierende Belege werden dabei Interviewauszüge zitiert, die als besonders eindrücklich oder typisch gesehen werden. Diese Vorgehensweise ist in der qualitativen Sozialforschung »ein wichtiges und meist auch gleich das einzige Mittel zur Dokumentation seiner [gemeint ist der Forscher] Aussagen « ${ }^{23}$.

Um der Komplexität der militärischen Medienbeziehungen gerecht zu werden, stützt sich die empirische Untersuchung auf einen theoretischen Ansatz, der die soziologische Akteur-, Institutionen- und Systemtheorie verbindet, also handlungsund systemtheoretische Erkenntnisse integriert, ohne die die moderne Gesellschaft konstituierende und für das Forschungsprojekt zentrale Ebene der (militärischen) Organisation zu vernachlässigen. Den theoretischen Ausgangspunkt liefert der sozialintegrative Ansatz des Soziologen Uwe Schimank ${ }^{24}$, den wir mit Überlegungen aus der Organisations ${ }^{25}$ und der Organisationskommunikationstheorie ${ }^{26}$ spezifiziert und für die empirische Forschung fruchtbar gemacht haben. In die theoretische Grundlegung einbezogen wurden darüber hinaus Ansätze aus der Public Relations-Forschung, der Militärsoziologie, der vergleichenden Politikforschung, der politischen Kommunikationsforschung sowie der komparativen Medien- und Journalismusforschung. Damit können die Beziehungen von Militär und Medien auf der Makro-Ebene (z.B. Wechselbeziehungen zwischen militärischer Öffentlichkeitsarbeit und Journalismus), der Meso-Ebene (Aufgaben, Leistungen, Strukturen und Bedingungen der medienbezogenen militärischen Kommunikation) und der Mi-

20 Die Leitfadeninterviews werden einer generalisierenden Analyse unterzogen (vgl. dazu Siegfried Lamnek, Qualitative Sozialforschung, Basel 2010, S. 368). Dabei »blickt man über das einzelne Interview hinaus, um zu allgemeineren (theoretischen) Erkenntnissen zu gelangen« (ebd.). Der Befragte wird dabei nicht als Einzelfall, sondern als Repräsentant einer Gruppe in die Untersuchung einbezogen (vgl. Horst Otto Mayer, Interview und schriftliche Befragung, München 2009, S. 38). »Die Darstellung der Ergebnisse geschieht [dann] aus einer theoretisch informierten Perspektive auf die empirisch generalisierten >Tatbestände`.« (Michael Meuser und Ulrike Nagel, 1997, S. 489, zit. in: Mayer, Interview und schriftliche Befragung, S. 55).

21 »Die zu einem bestimmten Zeitpunkt gegebenen Antworten der Befragten sind nicht einfach Produkt einer unabänderlichen Auffassung, Meinung oder Verhaltensweise, sondern sie sind prozesshaft generierte Ausschnitte der Konstruktion und Reproduktion von sozialer Realität." (Lamnek, Qualitative Sozialforschung - wie Anm. 20 -, S. $318 \mathrm{f}$.).

22 Vgl. ebd., S. 317.

23 Doris Bühler-Niederberger, Analytische Induktion als Verfahren der qualitativen Methodologie. In: Zeitschrift für Soziologie, 6 (1985), S. 475.

24 Vgl. u.a. Uwe Schimank, Theorien gesellschaftlicher Differenzierung, Opladen 1996, S. 241-269; Uwe Schimank, Organisationen: Akteurkonstellationen - Korporative Akteure - Sozialsysteme. In: Organisationssoziologie. Hrsg. von Jutta Allmendinger und Thomas Hinz, Wiesbaden 2002 (= Kölner Zeitschrift für Soziologie und Sozialpsychologie, Sonderheft 42), S. 29-54.

25 Vgl. u.a. Günter Endruweit, Organisationssoziologie, Stuttgart 2004.

26 Vgl. u.a. Anna Maria Theis-Berglmair, Organisationskommunikation. In: Öffentliche Kommunikation. Handbuch Kommunikations- und Medienwissenschaft. Hrsg. von Günter Bentele, Hans-Bernd Brosius und Otfried Jarren, Wiesbaden 2003, S. 565-575. 
kro-Ebene (handelnde Akteure mit ihren jeweiligen Erfahrungshintergründen) mit Hilfe eines theoretischen Ansatzes beschrieben werden ${ }^{27}$.

Unser Erkenntnisinteresse bezieht sich dementsprechend auf alle drei Ebenen militärischer Medienbeziehungen und ihre Verschränkung miteinander. In diesem Beitrag konzentrieren wir uns jedoch auf die Beschreibung der wesentlichen gesellschaftlichen und organisatorischen Veränderungen der militärischen Medienbeziehungen in Deutschland seit 1990. Im Kern interessieren wir uns dabei insbesondere für die Frage, welche Faktoren die Beziehungen der Bundeswehr zu den Medien hauptsächlich prägen. Sind es primär sicherheitspolitische Erwägungen? Welche Bedeutung kommt dem obersten Dienstherrn, also dem jeweiligen Verteidigungsminister zu? Und welche Rolle spielen organisationsspezifische Aspekte, etwa die Organisationsstruktur, die Personalzahl oder deren Qualifikation? Darüber hinaus analysieren wir in diesem Beitrag die Frage, welche Haltung hochrangige Offiziere der Bundeswehr gegenüber den Medien einnehmen. Hat sich die militärische Sicht auf Medien und Journalismus im Untersuchungszeitraum verändert? Oder gibt es so etwas wie eine Konstante in der soldatischen Wahrnehmung von Medien?

\section{Von der »Presseabwehr« zur aktiven Medienarbeit der Bundeswehr}

»Spaßeshalber hat man ganz früher [den Presseoffizier] Presseabwehroffizier [genannt], weil es immer nur darum ging, Schaden abzuwenden ${ }^{28}$ « Während des Kalten Krieges und in den ersten Jahren nach dem Ende der Ost-West-Konfrontation habe es als "schick « gegolten, wenn ein Truppenoffizier während seiner gesamten militärischen Laufbahn noch nie mit einem Journalisten gesprochen habe. Um die Bundeswehr sei ein »Schutzwall $\ll^{29}$ errichtet worden. Erklärt wird diese abwehrende Haltung von verschiedenen Gesprächspartnern mit einer damals vorherrschenden kritisch-simplifizierenden Berichterstattung. Die Medien hätten vor allem den »Gammeldienst bei der Bundeswehr« in den Mittelpunkt gerückt. Die deutschen Streitkräfte hätten deshalb »per se eigentlich immer einen schlechten Ruf [gehabt] [...], weil die uns immer alle unterstellt haben, wir taugen nichts, [...] wir kosten nur viel Geld « ${ }^{30}$. Die medienbezogene Kommunikation der Bundeswehr sei deshalb vor allem darauf ausgerichtet gewesen, Öffentlichkeitsarbeit mit Blick darauf zu betreiben, wie die Medienberichterstattung in der eigenen Organisation ankomme. Erst vor dem Hintergrund neuer kommunikativer Herausforderungen

27 Für eine detaillierte Darstellung des theoretischen Ansatzes vgl. Martin Löffelholz, Claudia Auer und Kathrin Schleicher, Organisationskommunikation aus sozial integrativer Perspektive. Die Verknüpfung von Akteur-, Institutionen- und Systemtheorie als Ausgangspunkt der PR-Forschung. In: Organisationskommunikation und Public Relations. Forschungsparadigmen und neue Perspektiven. Hrsg. von Ansgar Zerfaß, Lars Rademacher und Stefan Wehmeier, Wiesbaden 2012 (in Vorbereitung).

28 Offizier der Bundeswehr in einem Presse- und Informationszentrum (PIZ) der Bundeswehr, Leitfadengespräch 10, geführt im September 2010. Diese Einschätzung - inklusive des entsprechenden Begriffs - findet sich auch bei Bredow, Der Primat militärischen Denkens (wie Anm. 2), S. 64.

29 Presseoffizier PIZ (wie Anm. 28).

30 Ebd. 
- vor allem eines zwar nicht durchgängig großen, aber gleichwohl deutlich stärkeren medialen Interesses an den Auslandseinsätzen - sei, so die Gesprächspartner, erkannt worden, dass es unterschiedliche Bedürfnisse im Hinblick auf die interne und externe Kommunikation der Bundeswehr gebe:

»Wir haben immer geguckt, wie wirkt das auf uns intern, und wenn es intern gut war, haben wir gesagt, dann ist es auch gut für außen. Und ich glaub', da haben wir einen Perspektivwechsel mittlerweile in vielen [...] Bereichen vorgenommen, [so] dass wir jetzt sagen, wir müssen viel stärker darauf gucken, wie wirkt es außen ${ }^{31}$ «

Vor allem die zunehmende Beteiligung der Bundeswehr an Auslandseinsätzen war aus Sicht verschiedener Gesprächspartner für eine langsame Öffnung gegenüber den Medien verantwortlich. In den ersten Jahren sei Medienarbeit zwar noch durchgängig reaktiv betrieben worden - nach dem Motto: »Die Medien können mitkommen, aber wenn keiner nachfragt, ist auch gut, denn die Medien könnten negative Schlagzeilen bringen ${ }^{32}$. «Dennoch habe sich nach und nach die Erkenntnis durchgesetzt, dass über die Einsätze - vor allem über die deutsche Beteiligung an der internationalen Mission in Afghanistan - offen berichtet werden müsse. Allerdings erst vor kurzem habe es dabei einen "große[n] qualitative[n] Sprung [gegeben], Journalisten mit in den Einsätzen zu haben « oder sogar auf bewaffnete Patrouillen mitzunehmen: »[D]as hätten wir 2006 definitiv noch nicht gemacht, und das ist 2009/2010 Alltag gewesen ${ }^{33}$.«

Die nunmehr aktivere Medienarbeit der Bundeswehr ist nach Auffassung der Gesprächspartner auch in anderen Bereichen beobachtbar. So wurde 2010 beispielsweise die Online-Berichterstattung ausgeweitet. Meldungen würden deutlich früher ins Internet gestellt, darunter Sofortmeldungen, die beinahe täglich sogar kleinere Vorfälle nachvollziehbar machten. Über RSS-Feeds ${ }^{34}$ würden den Abonnenten (darunter viele Journalisten) aktuelle Meldungen angezeigt ${ }^{35}$. Insgesamt, so sagen mehrere Gesprächspartner, betrachte die Bundeswehr die Medien heute - vor allem als Folge der Auslandseinsätze - nicht als »Feind «, sondern als »Kooperationspartner « ${ }^{36}$. »[D]er Umgang mit Medien [erfolgt] ein Stückchen entkrampfter $[\ldots]$, nicht unvorsichtiger, aber entkrampfter ${ }^{37} . "$

Trotz dieser von den Gesprächspartnern beschriebenen langsamen Öffnung gegenüber den Medien herrsche in der Bundeswehr jedoch bei manchen Offizieren weiterhin ein gewisses Misstrauen gegenüber Journalisten. Einzelne folgten sogar weiterhin einer »Feindvorstellung [...] gegenüber den öffentlichen Medien: [...] die Presse immer als diejenigen zu sehen, die nur die Schlagzeile möchten, das Plakative, das Falsche ${ }^{38}$. Ein Interviewpartner beschreibt diese Skepsis gegenüber Medien als »etwas Systemimmanentes, dass sich militärische Hierarchie grundsätz-

Ebd.

Ebd.

Ebd.

RSS bedeutet »Really Simple Syndication« (oder »Rich Site Summary«) und ermöglicht den schnellen Online-Austausch von Inhalten.

35 Presse/-Infostab des BMVg, Leitfadengespräch 7, geführt im September 2010.

36 Offizier der Bundeswehr, Leiter eines PIZ der Bundeswehr, Leitfadengespräch 4, geführt im Februar 2011.

37 Offizier der Bundeswehr, Führungsstab, Leitfadengespräch 26, geführt im April 2011.

38 Ebd. 
lich zurückhält « ${ }^{39}$. Es sei »so etwas wie eine jahrhundertelange Kultur in öffentlichen Äußerungen [...] gegenüber Medien [...] nicht allzu sehr zur Verfügung [zu] stehen $\aleph^{40}$. Begründet wird diese Vorsicht mit den unterschiedlichen Systemlogiken von Militär und Medien: So müssten Streitkräfte, vor allem während gewaltsam ausgetragener Konflikte, auf die Geheimhaltung von Informationen achten. Demgegenüber stehe der Anspruch der Medien auf Informationsvermittlung und Informationstransparenz ${ }^{41}$.

Einzelne Gesprächspartner relativieren diesen Hinweis auf ein prinzipielles Konkurrenzverhältnis von Militär und Medien allerdings. Sie sehen die skeptische Zurückhaltung mancher Offiziere gegenüber Journalisten eher als Generationen-, Hierarchie- oder Zuständigkeitsproblem: »Gerade bei [...] hohen Generalen, Admiralen, die sind damit nicht aufgewachsen, und die haben immer so ein bisschen [...] noch das Gefühl, mit dem will ich nichts zu tun haben, das soll der Presseoffizier erledigen ${ }^{42}$. Zudem gäbe es unterschiedliche Auffassungen über den Umgang mit Medienvertretern zwischen den Bereichen der Bundeswehr, die im engeren Sinne für militärische Operationen verantwortlich, und jenen, die im Presse- und Informationsstab des BMVg (Presse/-Infostab) für die Kommunikation zuständig seien.

»Also naturgemäß ist es so, dass das Haus [das BMVg] am liebsten dicht hält, die Rollläden runter macht, also der militärische Bereich gerne hinter verschlossenen Türen arbeitet, mit der Presse nichts zu tun haben möchte. Wenn aber die Reputation des Hauses, des militärischen Bereichs in der Öffentlichkeit nicht mehr gegeben ist, dann heißt es, der Presse/-Infostab ist schuld dran, der versagt nun, weil er es nicht schafft, Reputation in der Öffentlichkeit herzustellen. Aber dass man dazu vielleicht die Tür auch öffnen [...] und neue Wege gehen müsste, dieses Verständnis ist nur sehr rudimentär vorhanden ${ }^{43}$."

Wie offen oder aber restriktiv die militärischen Medienbeziehungen in Deutschland konkret gestaltet werden, ist nach Einschätzung der Gesprächspartner stark von der Haltung des jeweiligen Verteidigungsministers abhängig. So sei zum Beispiel in der Amtsperiode des früheren Verteidigungsministers Jung (2006-2009) eher restriktiv agiert worden. Es habe auffällig weniger offizielle Pressetermine des BMVg bzw. der Bundeswehr in Berlin gegeben. Abgenommen hätte zudem die Bereitschaft militärischer Verantwortungsträger, an Hintergrundgesprächen mit Journalisten teilzunehmen ${ }^{44}$. Zurückgeführt wird diese restriktivere Phase militärischer Medienbeziehungen in Deutschland auf die Haltung und das Image des Verteidigungsministers:

»Natürlich ergibt sich dann, wenn jemand, wenn er der Presse nicht so gut gelitten ist, ständig auf Kritik in der Öffentlichkeit über die Medien stößt, [...] schon ein anderer Zugang zur Presse, als wenn, wie wir es jetzt [zur Amtsperiode des Verteidigungsministers zu Guttenberg] erlebt haben, dass jemand eben sehr positiv aufgenommen wird von der Presse ${ }^{45} . \ll$

Ebd.

40 Ebd.

41 Offizier der Bundeswehr im Presse/-Infostab des BMVg, Leitfadengespräch 8, geführt im September 2010.

42 Offizier der Bundeswehr, Kommandeur, Leitfadengespräch 23, geführt im April 2011.

43 Offizier Presse/-Infostab (wie Anm. 41).

44 Offizier der Bundeswehr in der AIK, Leitfadengespräch 6, geführt im Januar 2011. 
Ein den Medien zugewandter und von den Medien recht positiv beurteilter Minister stelle eine wichtige Voraussetzung für eine offenere Gestaltung der militärischen Medienbeziehungen dar:

»Ich glaube, wir hatten insofern eine erfolgreiche Pressearbeit machen können, weil wir einen ungeheuer offenen Minister hatten [...] Ich glaube, das war [...] und ist die halbe Miete für eine erfolgreiche Pressearbeit. Das hängt immer mit dem Minister zusammen, wie sehr er auch diese Offenheit will ${ }^{46}$."

Neben dem Verteidigungsminister kommt aus Sicht der Befragten insbesondere den militärischen Führern in Auslandseinsätzen eine prägende Rolle bei der Gestaltung der militärischen Medienbeziehungen zu: »Es ist [...] abhängig davon, wie [...] die verantwortlichen Kommandeure im Einsatz [...] mit dieser ganzen Thematik umgehen ${ }^{47}$.«

Die Beziehungen von Bundeswehr und Medien sind zusätzlich durch die von einzelnen Gesprächspartnern wahrgenommenen unterschiedlichen Kommunikationsziele der politischen und militärischen Führung gerade bei der Vorbereitung und Durchführung militärischer Einsätze im Ausland geprägt. Die Bundesregierung sei

»eigentlich immer darauf gerichtet [gewesen], im Inland möglichst wenig [zu] berichten über Vorbereitung und potenzielle Einsätze. Die Decke drauf zu halten, international die Kontakte zu machen - und irgendwann kamen dann die Entscheidungen. Die kamen dann meistens überraschend. Und dann musste immer alles ganz, ganz schnell gehen und zwar möglichst mit ganz wenig Mitteln und Kräften. Das konnte nicht im Sinne der militärischen Führung $\operatorname{sein}^{48}$.«

Wegen dieser Sprachlosigkeit politischer Instanzen, also der bewussten Zurückhaltung des Bundeskanzleramtes, des Bundesverteidigungsministeriums und parlamentarischer Gremien, die sich bis zur Amtsübernahme des ehemaligen Verteidigungsministers zu Guttenberg bemühten, den Afghanistaneinsatz aus der öffentlichen Diskussion herauszuhalten, sei die Bundeswehr unabsichtlich zu einem Sprachrohr der öffentlichen Legitimierung von Auslandseinsätzen geworden. Diese ureigentliche Aufgabe der Politik sei damit partiell auf die Bundeswehr übertragen worden. Doch mit der Erwartung, über militärische Öffentlichkeitsarbeit Entscheidungen von Bundestag und Bundesregierung zu rechtfertigen, sei die Bundeswehr überfordert ${ }^{49}$.

Neben einer eher reaktiven Beziehung des Militärs zur Politik beschreiben einzelne Gesprächspartner beispielhaft, wie die Bundeswehr ihre Interessen trotz des Primats der Politik durchaus auch aktiv mithilfe von Medien vertritt:

Offizier der Bundeswehr im Presse/-Infostab des BMVg, Leitfadengespräch 15, geführt im Februar 2011.

47 Offizier der Bundeswehr, Kommandeur, Leitfadengespräch 28, geführt im April 2011.

48 Offizier der Bundeswehr, Befehlshaber, Leitfadengespräch 16, geführt im Februar 2011.

49 Ebd.; zur Sprachlosigkeit politischer Akteure vgl. Martin Löffelholz und Kathrin Schleicher, Die Rolle der Medien: Vermittler oder Gestalter. In: Einsatz für den Frieden. Sicherheit und Entwicklung in Räumen begrenzter Staatlichkeit. Hrsg. von Josef Braml, Eberhard Sandschneider und Thomas Risse, München 2010 (= Jahrbuch Internationale Politik, 28), S. 398-403; Martin Löffelholz, Der »Kunduz-Fall« und die Medien. In: Loyal, 6/2010, S. 12-15; Martin Löffelholz, Weder strategisch noch modern. In: Loyal, 1/2010, S. 34-38. 
»[D]ie politische Führung [war] drauf und dran [...], von mir zu verlangen, mit relativ wenigen Soldaten in ein Einsatzgebiet in Afghanistan zu gehen, von dem ich wusste, dass die dortige Operation erstens gefährlich sein würde und zweitens mit den mir zugestandenen 200 Soldaten auf keinen Fall erfolgreich geführt werden konnte [...] Damit konnte ich auf dem Dienstweg wenig anfangen, weil die Glocken im Ministerium schlugen und die politischen Gesichtspunkte die militärischen überwogen. Das Thema wurde aber öffentlich, [...] und da hatte ich Kontakte, Hintergrundgespräche, in denen ich auch deutlich gemacht habe, welche großen Sorgen ich habe [...] Letztlich wurde diese Operation so nicht durchgeführt ${ }^{50}$.

Die Beziehungen der Bundeswehr zu den Medien haben sich nach 1990 aus Sicht verschiedener Gesprächspartner jedoch nicht nur gewandelt, weil sich die deutschen Streitkräfte stärker zu einer Einsatzarmee entwickelt haben und dadurch neue kommunikative Herausforderungen entstanden seien. Mit dem Umzug der Bundesregierung von Bonn nach Berlin habe sich eine andere Kultur des Hauptstadtjournalismus entwickelt - mit erheblichen Konsequenzen für die Gestaltung der militärischen Medienbeziehungen. Vertreter von BMVg und Bundeswehr stünden seither einem »Heer der Journalisten ${ }^{51}$ gegenüber. Aus Sicht eines Gesprächspartners gab es

»unter den Journalisten in Bonn so eine verschworene Gemeinschaft, dass man sich irgendwo treffen konnte. Und das wurde nicht sofort irgendwo publik. Mit dem Umzug nach Berlin, [...] man kann nicht mehr ganz sicher sein, ob jeder Journalist, der bei einem Sender, einer kleinen Zeitung oder sonst wo tätig ist, auch die Regeln der Bundespressekonferenz einhält [...] Aber wenn da zu einer Pressekonferenz eingeladen ist und da tauchen dann so alle möglichen Journalisten auf, die man gar nicht kennt [...], ist man sehr viel vorsichtiger geworden, irgendwelche Äußerungen zu machen. Also dahingehend [bezüglich der Ausgestaltung der Bundespressekonferenz] hat sich die Presse-und Öffentlichkeitsarbeit schon mit dem Umzug nach Berlin erheblich gewandelt ${ }^{52}$.«

Der Journalismus der Berliner Republik ist aus Sicht der Befragten zudem durch einen deutlich größeren Konkurrenzdruck und einer stärkeren Boulevardisierung der Berichterstattung gekennzeichnet.

»Durch den hohen Konkurrenzdruck tritt auch eine höhere Kontaktzahl ein bei den Journalisten, die müssen wesentlich mehr Themenfelder beackern, gehen nicht mehr in die Tiefe. Und was man beobachten kann: einer schreibt von den anderen ab. Es gibt keine Journalisten mehr, gerade im sicherheitspolitischen Bereich, die das Thema sicherheitspolitisch tiefgründig abarbeiten. Sondern es wird oberflächlich abgearbeitet, was das Fachliche angeht. Ansonsten konzentriert man sich auf das Geschehen Boulevard-Mitte oder Berliner S-Bahn-Ring. Also was so in Berlin Mitte vor allem [...] auf dem politischen Boulevard stattfindet, das ist für die sicherheitspolitischen Journalisten das Entscheidende. Also, wie kann man dem Minister [dem ehemaligen Verteidigungsminister 
Franz-Josef Jung] oder anderen einen mitgeben, aber es geht nicht mehr um die Sache ${ }^{53} \cdot \ll$

Neben der aus Sicht der Befragten wenig tiefgründigen Berichterstattung werden die militärischen Medienbeziehungen zudem geprägt von dem zumeist geringen medialen Interesse an Sicherheitspolitik:

»Also wenn Sie Sendungen sehen, gerade auch aus dem Bereich des Einsatzgeschehens, wo man eigentlich sagt, gut, das ist nun das, was die Leute interessieren sollte. Das interessiert sie in letzter Konsequenz aber auch nicht. Dann werden die Sendungen um 23.30 Uhr eingestellt oder um 0.10 Uhr oder sonst irgendwas. Eigentlich ist Bundeswehr, Sicherheitspolitik, eine vollkommene Nischenveranstaltung ${ }^{54}$.«

Um das in Zukunft zu ändern, also mehr öffentliche Aufmerksamkeit für die Bundeswehr zu generieren, sehen einzelne Gesprächspartner als mögliche Perspektive, die Zusammenarbeit mit Medien bei fiktionalen Formaten auszuweiten:

»Wir können natürlich [...] für anderthalb Millionen Euro [...] einen 20-Sekunden Spot machen und den zwei bis drei Wochen in verschiedenen Vorabendserien präsentieren, in der Hoffnung, dass Menschen das sehen und sagen, Bundeswehr ist toll [...] Wir können aber auch für dieses Ding [beispielsweise eine Fernsehserie], was uns so gut wie nichts gekostet hat, weil der Sender [...] die Kosten übernommen hat, können wir natürlich auch Stück für Stück Millionen von Menschen erreichen ${ }^{55}$ «

\section{Organisatorische Charakteristika der Medienbeziehungen der Bundeswehr}

Als zentrale Hindernisse einer offenen und flexiblen Medienarbeit nennen verschiedene Befragte insbesondere die enorme Komplexität der Organisationsstruktur sowie die vergleichsweise starren und strikt hierarchisch aufgebauten Strukturen der Bundeswehr. So verhinderten einzuhaltende Dienstwege, dass in entsprechenden Situationen hinreichend schnell reagiert werden könne:

»Aber hier ist es so, dass vor jedem Bild, was da gemacht wird, die ganze militärische Hierarchie davor steht. X Generäle, die dann sagen, nein, wenn das Bild der und der Abgeordnete dann sieht, dann [...] muss ich mich dann wieder rechtfertigen ${ }^{56}$.«

Offizier Presse/-Infostab (wie Anm. 41); zur Berichterstattung über sicherheitspolitische Themen vgl. Martin Löffelholz, Die Kommunikation von Krisen: Medien zwischen strategischer Offentlichkeitsarbeit und ambivalenten Publikumsinteressen. In: Jahrbuch Öffentliche Sicherheit 2010/11. Hrsg. von Martin H.W. Möllers und Robert Chr. van Ooyen, Frankfurt a.M. 2011, S. 31-39.

55 Offizier AIK (wie Anm. 44); zur Mediennutzung in Krisen vgl. Volker Gehrau und Alexander Görke, Alarm im Wohnzimmer. Wie sich die Mediennutzung in Krisenzeiten ändert. In: Kriegs- und Krisenberichterstattung. Ein Handbuch. Hrsg. von Martin Löffelholz, Christian F. Trippe, und Andrea C. Hoffmann, Konstanz 2008, S. 292-296. 
Die komplexe Organisationsstruktur führe zudem dazu, dass es zu viele interne Akteure gäbe, die für die Beziehungen zu den Medien verantwortlich seien. Abgesehen von dem Presse/-Infostab des BMVg, der eine leitende und koordinierende Funktion ausübe, seien die Presse- und Informationszentren (PIZ) der Teilstreitkräfte für die detaillierte Ausgestaltung der Medienbeziehungen zuständig. Zudem verfügten die sich darunter befindenden militärischen Hierarchieebenen ebenfalls über entsprechende (kleinere) Kommunikationsabteilungen. Die komplexen organisatorischen Strukturen der militärischen Medienarbeit werden nicht zuletzt im Internet deutlich. Zwar hat sich die Bundeswehr vor einigen Jahren um eine Vereinheitlichung ihres Internetauftritts bemüht. Bis heute findet sich jedoch - neben der Hauptseite (www.bundeswehr.de) - eine Vielzahl von Unterseiten verschiedenster militärischer Subbereiche (so kommt man zum Internetauftritt des Heeres über: www.deutschesheer.de). Durch die Aufsplitterung der Kommunikationsarbeit würden, wie einzelne Gesprächspartner anmerken, nicht selten Partikularinteressen des eigenen Organisationsbereiches oder des Kommandos vertreten, statt eine einheitliche Botschaft zu vermitteln: »So und jetzt wissen Sie auch, warum wir keine Marke Bundeswehr haben. « Notwendig sei aber, »mehr Bundeswehr [zu] denken, [...] weg von der Vermittlung von hierarchischen Strukturen hin zum Vermitteln von Fähigkeiten « ${ }^{57}$.

Die Zersplitterung in verschiedene Organisationsbereiche erschwere Medienvertretern zudem, den richtigen Ansprechpartner in der Bundeswehr zu finden. Außerdem werde dadurch die angestrebte `One-Voice-Policy behindert. Zwar bemühe sich der Presse/-Infostab des BMVg um die Kontrolle von Informationen in der eigenen Truppe mit Hilfe von Sprachregelungen. Diese Kontrolle gelänge jedoch nicht immer. So würden sich beispielsweise manche Kommandeure im Auslandseinsatz nicht immer an die entsprechenden Sprachregelungen halten.

»Und da gibt es die andere Facette, eine gewisse Korona unter höheren Offizieren an Narzissten, die das offizielle Organ, also den Presse-/Infostab, in seiner Funktion nicht unterstützen, aber dann hintenrum sich gut finden, wenn sie mit dem Journalisten Kaffee trinken gehen, die Papierchen durchstechen und sich daran weiden, wie sich die Blödmänner und Blödfrauen vom Presseund Infostab dann abmühen, diese oder jene Welle wieder zu verflachen ${ }^{58}$ «"

Die Komplexität der Organisationsstruktur könne zudem zu internen Leitungskonflikten führen, die die Beziehungen zu Medien durchaus negativ beeinflussen könnten. Beispielsweise sei ein Leiter der Informationsarbeit (LdI) auf Divisionsebene sowohl dem Divisionskommandeur, als auch dem PIZ der jeweiligen Teilstreitkraft unterstellt. Damit muss er unterschiedlichen Ansprüchen gerecht wer$\operatorname{den}^{59}$.

Die oben beschriebenen veränderten kommunikativen Ansprüche an die Bundeswehr führten im Laufe der letzten beiden Jahrzehnte immer wieder zu organisatorischen Veränderungen der Medienarbeit. So waren im Streitkräfteamt die Presse- und Öffentlichkeitsarbeit (in Meckenheim), Medienzentrale (St. Augustin) und Nachwuchswerbung (Bonn-Duisdorf) zunächst regional und liegenschaftsmäßig voneinander getrennt. Um eine höhere Effizienz (Personaleinsparung) und

57 Leiter PIZ (wie Anm. 36).

58 Offizier Presse/-Infostab (wie Anm. 41).

59 Offizier der Bundeswehr, Operative Information, Leitfadengespräch 1, geführt im August 2010. 
Effektivität zu erreichen, wurden diese Teilbereiche Mitte der 1990er Jahre räumlich unter dem Dach der Informations- und Medienzentrale der Bundeswehr in St. Augustin zentralisiert ${ }^{60}$. Obgleich in manchen Bereichen der militärischen Medienarbeit Personal abgebaut wurde, halten die Gesprächspartner insgesamt fest, dass den gestiegenen Ansprüchen an die Kommunikationsarbeit personell durchaus Rechnung getragen wurde:

»Also am Anfang [2000] war es so, dass man eigentlich eine ganz kleine Pressezelle [im PIZ Streitkräftebasis] hatte [...] Und das war eigentlich ausgelegt rein auf reaktive Pressearbeit und ein bisschen Mitarbeiterkommunikation [...] Es ist dann im Laufe der Zeit immer mehr an Fähigkeiten dazu gekommen, und das PIZ hat [...] einen enormen Aufwuchs erfahren, rein vom Personal her, und hat sich dann letzten Endes in vier Dezernate gegliedert ${ }^{61}$."

So sei es letztlich erst möglich geworden, nicht bloß auf Medienberichterstattung $\mathrm{zu}$ reagieren, sondern auch aktiv auf diese einzuwirken und eigene Themen zu lancieren ${ }^{62}$.

Allerdings werde diese eigentlich begrüßenswerte Personalsituation konterkariert, weil zugeteilte Kräfte beim Sport, bei einer Weiterbildung oder im Urlaub seien, sich auf einen Auslandseinsatz vorbereiteten oder sich für mehrere Monate im Auslandseinsatz befänden. Die Presse- und Informationszentren der Bundeswehr arbeiteten daher oftmals mit deutlich weniger Personal, als ihnen auf dem Papier zur Verfügung stehe ${ }^{63}$. Mit dem Personalmangel in Deutschland würden beispielsweise die Presseoffiziere im Auslandseinsatz konfrontiert - mit unmittelbaren Folgen für die militärischen Medienbeziehungen, denn an Wochenenden würden bundeswehrintern oftmals die richtigen Ansprechpartner fehlen. Die Bundeswehr sei eine »Montag-bis-Freitag «-Armee: »Das ist ein erheblicher unprofessioneller Umgang mit der Einsatzrealität ${ }^{64}$ «

Die Medienarbeit in Auslandseinsätzen werde darüber hinaus behindert, weil die zivile Wehrverwaltung wenig Verständnis für die Bedürfnisse der Truppe im Einsatz habe. So seien beispielsweise die Antragsverfahren zur Einstellung einheimischer Übersetzer, die für die Medienarbeit dringend benötigt würden, zu kompliziert, oder es fehlten schlicht Ausrüstungsgegenstände:

»Wir müssten raus, raus zur Zielgruppe, müssen uns mit den Afghanen unterhalten, [...] wir müssten die Lebensrealität kennenlernen, wir müssen für die [Bundeswehr] Medienarbeit machen. Können aber nicht raus, weil die Fahrzeuge fehlen beziehungsweise bürokratische Regularien das einfach unmöglich machen ${ }^{65}$.«

Neben einem quantitativen Personalausbau ist im Untersuchungszeitraum, wie mehrere Gesprächspartner anmerken, ein Wandel bei der Qualität des eingesetzten Personals im Hinblick auf Medien- und Kommunikationskompetenzen zu verzeichnen. Während ein Großteil der im Bereich Presse- und Öffentlichkeitsarbeit tätigen Soldaten noch bis Anfang der 1990er Jahre versuchte, sich im Do-it-yourself-Verfahren entsprechende Qualifikationen anzueignen, begann mit der Grün-

Offizier der Bundeswehr, Streitkräfteamt, Leitfadengespräch 24, geführt im März 2011. Presseoffizier PIZ (wie Anm. 28).

Ebd.

Ebd.

Offizier Operative Information (wie Anm. 61).

Ebd. 
dung der AIK die strukturierte Vermittlung notwendiger Kompetenzen, um das militärische Personal besser für die Medienarbeit zu qualifizieren und damit in einen Dialog mit der Öffentlichkeit treten zu können ${ }^{66}$. Erste Versuche einer systematischeren Schulung gab es zwar schon vor Gründung der AIK, aber »damals hat man sich so [...] herangetastet [...] Konkret war ich mal über einige Wochen in München an der deutschen Journalistenschule ${ }^{67}$. « Manche Presseoffiziere wurden auch »zu Zeitungen, zu Rundfunksendern, zu Fernsehsendern [abgestellt], um [...] die mal sehen zu lassen, wie wird eigentlich Journalismus gemacht ${ }^{68} \cdot$ «

Die AIK bemüht sich seit ihrer Gründung um eine stete Anpassung ihrer Ausbildungsinhalte an veränderte kommunikative Ansprüche. So werden seit 2005 zum Beispiel Einzelmedientrainings für Kommandeure im Auslandseinsatz angeboten, denn »Medienarbeit [ist] eigentlich Kommandeursaufgabe ${ }^{69}$. Im Laufe der Jahre hat sich dieser Bereich zu einer zentralen Säule im Ausbildungsportfolio der AIK entwickelt:

»[W]ir haben das als kleines Pflänzchen begonnen. Heute ist das Einzelmedientraining für Kommandeure in Afghanistan eine ausgesprochene Selbstverständlichkeit [...] - also nicht nur Afghanistankommandeure, [...] aber auch sonst für wichtige Generale, Admirale, aber auch zivile Vertreter dieser Ebenen. Das [...] hat sich gewaltig entwickelt. Wo man eben gesagt hat, [...] die Bedeutung der Medienarbeit ist gewachsen und durch diese Betroffenheit der verantwortlichen Dienststellenleiter, Kommandeure hat man [...] dem [...] Rechnung getragen ${ }^{70}$ « Das Bemühen um eine fundierte Ausbildung für Kommunikationsverantwortliche kollidiere jedoch mit der geringen Attraktivität kommunikationsbezogener Laufbahnen in der Bundeswehr. So gebe es keine kohärente militärische Laufbahn für die Informationsarbeit: Ein Presseoffizier, der Berufsoffizier werden möchte, stiege oftmals nicht zum Leiter der Informationsarbeit auf, sondern würde aus seinem Bereich abgezogen und in eine andere Verwendung oder in einen Auslandseinsatz geschickt: »Und der dann eben nicht die Gewissheit hat, nach diesen drei Jahren wieder in die Informationsarbeit zu kommen ${ }^{71}$."

Auswirkungen auf die Gestaltung der Medienbeziehungen der Bundeswehr haben darüber hinaus aus der Sicht vieler Gesprächspartner vor allem technologische Entwicklungen. Neue Informations- und Kommunikationstechnologien vereinfachten die Informationsrecherche, ermöglichten die multimediale Veröffentlichung von Informationen im Internet und böten somit die Chance, die Bevölkerung direkt zu erreichen, sofern die entsprechenden Internetauftritte hinlänglich attraktiv seien. Eingeschränkt würden diese Möglichkeiten allerdings durch die begrenzte Verfügbarkeit von Internetzugängen an den Arbeitsplätzen oder die Nichtverfügbarkeit internetfähiger Mobiltelefone:

"Selbst mir als Leiter ist es bisher nicht gelungen, dienstliche Geräte zu bekommen, mit denen ich unabhängig vom Ort internetfähig bin. Was mache ich? Ich mache das mit meinem [privaten] iPhone. Die Informationsarbeit ist [aus Sicht kommunikative Strategie (wie Anm. 1).

67 Befehlshaber (wie Anm. 48).

68 Offizier Streitkräfteamt (wie Anm. 62).

69 Kommandeur (wie Anm. 42).

70 Ebd.

71 Offizier der Bundeswehr im Auslandseinsatz, Leitfadengespräch 2, geführt im August 2010. 
der Leiter der PIZen] nicht auf [...] Arbeitszeit beschränkt. Information werden gewünscht oder müssen gegeben werden unabhängig von der Tageszeit: 24/7. So es ist trotz Einhaltung aller Schutzmechanismen möglich, dass ich mich einklinken kann von jedem Ort in mein System. Technisch [ist das] kein Problem, [...] [es] läuft [aber] nicht. Das ist wirklich sehr ärgerlich. Wir reden hier nur von Geld ${ }^{72}$ «

Der Einsatz sogenannter Social Media werde zudem behindert, weil das IT-System der Bundeswehr (»Herkules«) vergleichsweise unflexibel sei:

»Wir haben den Herkules-Vertrag, der drückt uns die Kehle zu. Wir sind bald nonop, not operational [...] Das ist sehr ärgerlich und behindert die Arbeit, dass meine Leute und alle anderen, die da mitspielen, eine sehr, sehr begrenzte technische Möglichkeit haben, um geeignet mitzuspielen ${ }^{73}$."

Denn in das System könnten weder Weblogs, noch Wikis, noch weitere interaktive Medien eingebaut werden. Das sei einer der Gründe, warum die Bundeswehr nunmehr einen eigenen Youtube-Kanal aufgebaut habe und zudem die im Internet zugängliche Fotoplattform Flickr nutze:

»Dieser Einstieg und die intensivere Nutzung der elektronischen Medien [...], das ist was vollkommen Neues, was vor zwei Jahren zwar gewünscht wurde von uns. Aber so nicht absehbar war, dass dort eine Bereitschaft gewesen wäre, das so umzusetzen ${ }^{74}$ «

Erst mit dem Wechsel zu einer offensiveren Medienarbeit unter Verteidigungsminister zu Guttenberg sei der Einstieg in diese neuen Kommunikationstechnologien möglich geworden.

\section{Fazit}

Veränderungen im Mediensystem, ein anderes sicherheitspolitisches Umfeld, die nunmehr langjährige Beteiligung an militärischen Einsätzen im Ausland, der Umbau der deutschen Streitkräfte zu einer Einsatzarmee - diese grundlegenden Veränderungen haben zu einer langsamen, aber gleichwohl grundlegenden Annäherung der Bundeswehr an die Medien geführt. Die Skepsis gegenüber den Medien, die die militärischen Medienbeziehungen noch Anfang der Neunziger Jahre prägte, ist einem Verständnis von Medien als Kooperationspartner gewichen. Begleitet wird dieser Wandel von einem professionelleren Umgang mit Journalisten und einer in jüngster Zeit beobachtbaren aktiveren Gestaltung der militärischen Medienbeziehungen. Diese Entwicklungen können freilich nicht als lineare Prozesse verstanden werden. Nach wie vor finden sich in der Bundeswehr Tendenzen einer eher ablehnenden Haltung gegenüber den Medien und ihren Vertretern. Wie offen oder wie restriktiv die Medienbeziehungen im Einzelnen gestaltet werden, hängt in Deutschland offenbar in besonderer Weise von der Haltung des amtie-

72

73

Offizier der Bundeswehr, Leiter einer Fachabteilung, Leitfadengespräch 18, geführt im März 2011. 
renden Verteidigungsministers zu den Medien sowie den Vorstellungen der jeweils für bestimmte Einsätze verantwortlichen militärischen Führung ab.

Die besondere Relevanz des Verteidigungsministers beruht nicht nur auf seiner Rolle als oberster Dienstherr aller Bundeswehrangehörigen, sondern zudem auf der funktionalen Ausrichtung und strukturellen Einbindung des Presse- und Informationsstabes in das BMVg. Der Stab muss sowohl die Initiativen der politischen Leitung (sowie den Verteidigungsminister als Person), als auch die Aktivitäten der Bundeswehr in die Öffentlichkeit kommunizieren. Der Leiter des Stabes ist gleichzeitig Sprecher des Verteidigungsministers, wodurch die Priorität auf dessen Person bzw. Politik gelegt wird. Bei einer eher defensiven Haltung des jeweiligen Verteidigungsministers führen die starke strukturelle Stellung des Stabes und seine besondere funktionale Ausrichtung nahezu unmittelbar zu eher restriktiven militärischen Medienbeziehungen. Allerdings versuchen manche Offiziere gelegentlich dennoch, wie oben fallbeispielhaft verdeutlicht, Medien einzuspannen, um eigene (allerdings in der Regel kaum grundsätzliche Aspekte berührende) Interessen zu verfolgen, die sie ansonsten in der militärischen Hierarchie kaum durchsetzen könnten.

Zusätzlich wird das Verhältnis der Bundeswehr zu den Medien von verschiedenen weiteren Aspekten geprägt, die sich vor allem auf die Organisation der militärischen Medienbeziehungen beziehen. Neben der Komplexität der Organisationsstruktur werden die Medienbeziehungen der Bundeswehr maßgeblich von dem verfügbaren Personal und seinen Qualifikationen beeinflusst. Insbesondere die Aggregation der Aussagen der Befragten spricht dafür, dass die von ihnen beschriebenen Probleme auf faktische Defizite der Organisation militärischer Medienbeziehungen verweisen. Die Gründung der Akademie der Bundeswehr für Information und Kommunikation und der Ausbau der Qualifizierungsmaßnahmen sind Indizien für den Relevanzgewinn der militärischen Medienbeziehungen. $\mathrm{Ob}$ damit das nicht immer große Selbstwertgefühl der für Medien zuständigen Bundeswehrangehörigen gestärkt werden kann, muss offen bleiben. Es ist jedoch davon auszugehen, dass die in dem Beitrag beschriebenen Anforderungen an die militärische Medienarbeit in Zukunft weiter steigen werden, auch durch den Wandel der Bundeswehr von einer Wehrpflicht- zu einer Berufsarmee.

\section{Abstract}

Since the 1990s, the German Bundeswehr increasingly opened up to the media. This can be mainly traced back to the growing number of out of area missions in conjunction with new communication needs. In the beginning of the 1990s, military media relations were generally conducted in a reactive manner, whereas in the subsequent years, they were actively shaped. How open or restrictive media relations were conducted in detail is depending upon a complex combination of factors. As such, military media relations are shaped for example by the different systems' logics of the military and the media, by the complex organizational structures of the German armed forces or by the available personnel and its qualifications. 